\title{
Mammalian microRNA: an important modulator of host-pathogen interactions in human viral infections
}

\author{
Chet Raj Ojha ${ }^{*}$, Myosotys Rodriguez ${ }^{1}$, Seth M. Dever ${ }^{1}$, Rita Mukhopadhyay ${ }^{2}$ and Nazira El-Hage ${ }^{1}$
}

\begin{abstract}
MicroRNAs (miRNAs), which are small non-coding RNAs expressed by almost all metazoans, have key roles in the regulation of cell differentiation, organism development and gene expression. Thousands of miRNAs regulating approximately $60 \%$ of the total human genome have been identified. They regulate genetic expression either by direct cleavage or by translational repression of the target mRNAs recognized through partial complementary base pairing. The active and functional unit of miRNA is its complex with Argonaute proteins known as the microRNAinduced silencing complex (miRISC). De-regulated miRNA expression in the human cell may contribute to a diverse group of disorders including cancer, cardiovascular dysfunctions, liver damage, immunological dysfunction, metabolic syndromes and pathogenic infections. Current day studies have revealed that miRNAs are indeed a pivotal component of host-pathogen interactions and host immune responses toward microorganisms. miRNA is emerging as a tool for genetic study, therapeutic development and diagnosis for human pathogenic infections caused by viruses, bacteria, parasites and fungi. Many pathogens can exploit the host miRNA system for their own benefit such as surviving inside the host cell, replication, pathogenesis and bypassing some host immune barriers, while some express pathogen-encoded miRNA inside the host contributing to their replication, survival and/or latency. In this review, we discuss the role and significance of miRNA in relation to some pathogenic viruses.
\end{abstract}

Keywords: microRNA, miRISC, Pathogens, Gene regulation

\section{Background}

Recent advancements in genomics and proteomics have shown that out of roughly half of the human genome which is transcribed into RNA transcripts, about $2 \%$ is translated into the corresponding amino acid sequences [1]. The remaining $98 \%$ of RNA transcripts are collectively known as non-coding RNAs (ncRNA) which may be divided into small non-coding RNA (sncRNA) or long non-coding RNA (lncRNA) [1, 2]. MicroRNAs (miRNAs) are endogenous small non-coding RNAs regulating gene expression in almost all metazoans [3]. In spite of coding for any proteins, miRNAs carry different information and execute different functions [4]. They regulate gene expression either by complete cleav-

\footnotetext{
* Correspondence: cojha001@fiu.edu

'Department of Immunology, Herbert Wertheim College of Medicine, Florida International University, Florida, USA

Full list of author information is available at the end of the article
}

age or by translational repression of the target mRNAs $[3,5,6]$ It has been speculated that approximately $30-60 \%$ of the human coding genome is regulated by thousands of miRNAs with diverse targets $[7,8]$.

The exciting avenue of miRNA was unraveled in 1993 by the finding that Lin-4, a heterochronic gene previously recognized for its role in regulating the temporal sequence of events involved in Caenorhabditis elegans (C. elegans) larval development to adult form, regulates the process by synthesizing a pair of small RNAs rather than coding for a protein [9]. Two small Lin-4 RNA transcripts containing complementary sequences to a repeating sequence element within the 3 '-untranslated region (3' UTR) of another mRNA (Lin-14) were identified in C. elegans [10]. The finding led to the prediction of a type of RNA-RNA binding and interaction which down-regulates the translation of the target mRNA [4]. Subsequently, the second miRNA (Let-7) with a similar function in the late development of larva was discovered 
in the same organism [11]. The names for miRNAs are assigned by using the prefix "miR" preceding a unique identification numeric (e.g., miR-1, miR-2 etc.). To make species specific, few letters from the name of the organism are added before miR (e.g.; hsa for Homo sapiens, $\mathrm{mmu}$ for mus musculus, rno from Rattus norvegicus, ath for Arabidopsis plant etc.) [12]. The genes coding for miRNAs are named by capitalization (e.g., MIR-), hyphenation and italicization (e.g., mir-) in accordance with the conventions for the particular organism [12, 13]. Currently, 35,828 total and 2588 human encoded mature miRNAs are registered in miRNA database (http://www.mirbase.org/) $[14,15]$.

With the expanding body of research on miRNAs in relation to important biological processes, their crucial role as regulators of cell differentiation, proliferation and growth, intracellular dynamics, and apoptosis has been established [3, 9]. De-regulated miRNA expression leads to human pathologies including cancer, cardiovascular disease, liver disorders, immunological dysfunction, and metabolic syndromes [5, 9]. There are a growing number of reviews on miRNAs and their role in the aforementioned disorders and some individual pathogenic infections. With this review we present the current understanding of miRNAs and their role in some viral pathogens.

\section{Biosynthesis and mode of action of miRNA}

Genes encoding several identical or similar miRNAs are generally located as a cluster in the genome, where they might be expressed simultaneously [16] or individually depending on the tissue types [17]. In mammals, RNA polymerase II transcribes the gene into a long transcript known as a primary miRNA (pri-miRNA) consisting of single or multiple hairpin structures (Fig. 1) [13, 18-21]. Pri-miRNA is trimmed into an approximately 70 nucleotide long hairpin structure known as pre-miRNA by Drosha complex $[22,23]$. The resulting pre-miRNA with a $5^{\prime}$ phosphate overhang and a 2 nucleotide 3 ' overhang is recognized by exportin-5 [24] and is transported out of the nucleus $[18,25,26]$. Once released in the cytoplasm, a specialized multi-domain ribonuclease III enzyme known as Dicer excises the pre-miRNA to remove the loop structure leaving the remaining miRNA duplex with a 2 nucleotide $3^{\prime}$ overhang $[18,27,28]$. Some functional miRNA such as miRtrons and Simtrons do not undergo the canonical process for their maturations $[29,30]$. In miRtron biogenesis, Drosha cleavage is substituted by splicing of intronic hairpin structures, which is followed by maturation through dicing [31]. Simtron is synthesized by a pathway that involves only Drosha but does not require DGCR8, Dicer, Ago2 or XPO5 for its further processing [32]. After Dicer cleavage and unwinding of the two strands of miR-miR duplex, one strand (anti-sense strand) of the resulting miRNA-miRNA duplex is loaded onto Argonaute proteins and miRNA-induced silencing complex (miRISC) is generated [3, 33].

miRNA regulates gene expression typically targeting and binding to the seed map site in 3 '-untranslated region ( $3^{\prime}$-UTR) of protein coding target messenger RNA (mRNA) leading to degradation or translational repression of the gene [18, 34]. However, miRNAs can also target to the sites other than $3^{\prime}$ UTR, such as $5^{\prime}$ UTR and the coding regions of mRNA and lead to translational repression $[35,36]$. In mammal, perfect complimentary affects the stability and triggers tailing and $3^{\prime}$-to- $5^{\prime}$ trimming of miRNA [37]. It has been reported that some miRNAs can also mediate up-regulation of genes and that the genetic down-regulation mediated by miRNA can be reversible [38]. The upregulating miRNAs most likely direct the association of regulatory proteins complexes (Argonaute protein and fragile $\mathrm{X}$ mental retardation-related protein 1 (FXR1)) with AU-rich elements (AREs) in the 3'UTR of the mRNA, leading to activation of AREs as a translation signal [39]. Moreover, interaction of miRNA at 5'UTR can also trigger activation of translation [35].

\section{Role of miRNA in viral infections}

miRNAs play a crucial role in mounting an immune response against microbial infections caused by viruses, bacteria, parasites and fungi [40]. Nevertheless, many microorganisms have been shown to modulate the expression of several host miRNAs either to facilitate their own replication, survival and pathogenesis or for some unknown functions [30, 41-43]. Restriction of viral replication by RNA interference (RNAi) either by miRNA or siRNA in human cells is still a controversial issue [44]. Most of the human viruses when in their acute and replicative stages are thought to be resistant to endogenous RNAi mediated by miRNAs [44]. However, strong evidences to prove the role of miRNAs in restricting or promoting the replication and human pathology of viruses such as Hepatitis $B$ and $C$ as well as other viruses have been found $[45,46]$ Many of the cellular miRNAs affect viral replication either directly by binding to the viral genome or indirectly by targeting host factors related to replication [8].

Virus-encoded miRNAs (vmiRNA) identified in virusinfected human cells and other mammalian hosts significantly influence viral replication and disease progression by modulating viral as well as host cellular mRNA $[41,47]$. The first vmiRNA identified in humans was that encoded by the Epstein-Barr virus and subsequently, other members of herpesvirus, polyomavirus and adenovirus families were found to express vmiRNAs [41]. More than 200 vmiRNAs have been reported so far [47]. Although vmiRNAs encoded by RNA viruses are very rare, 


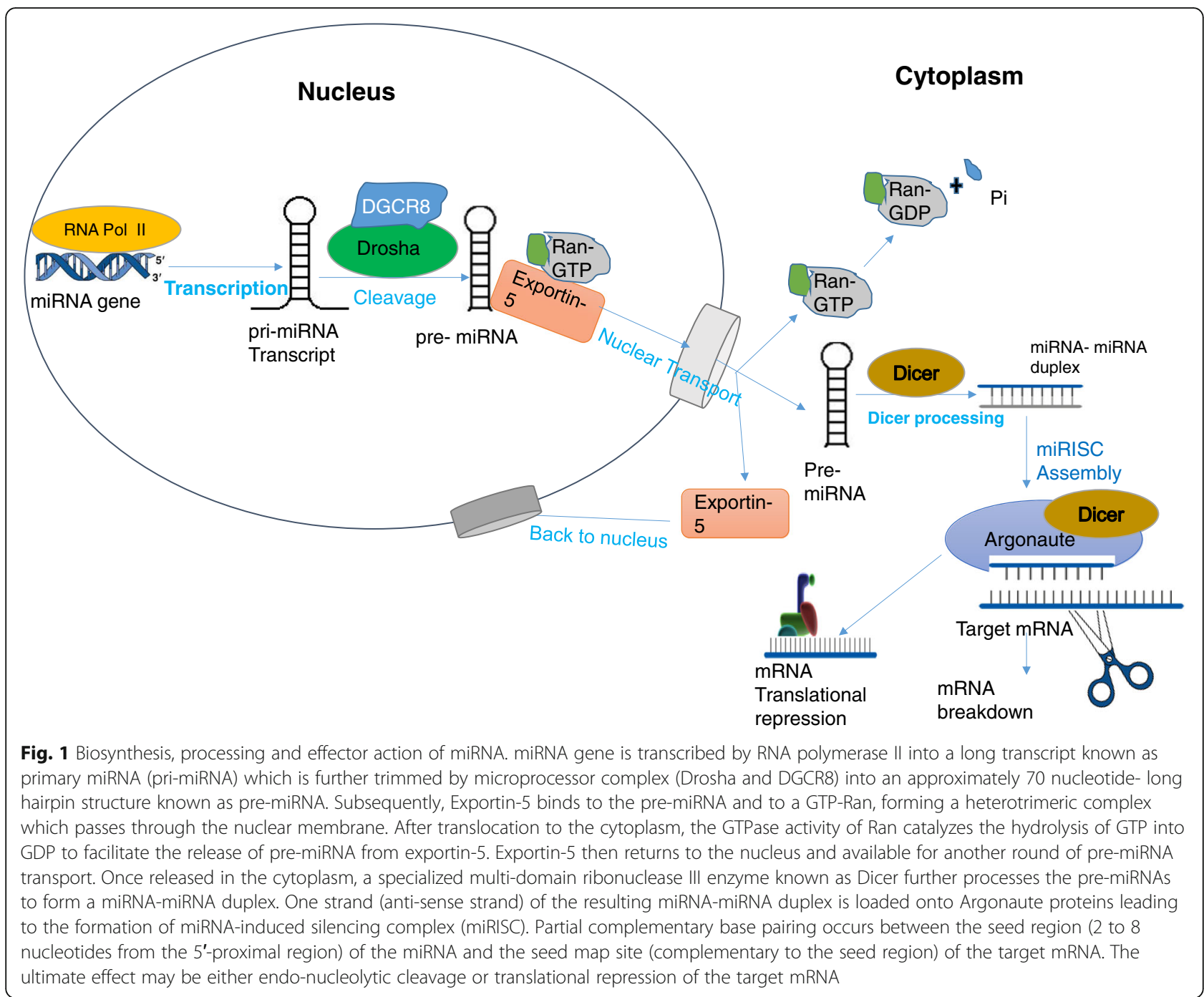

recently bovine leukemia virus has been found to encode a cluster of miRNAs transcribed by RNA Polymerase III, which is identical to human miR-29 [48]. Virus encoded miRNAs are also reported in culture cells as well as in cattle infected with bovine foamy virus (BFV), a member of retrovirus family and spumavirus subfamily [49]. Avian leukosis virus subgroup J (ALV-J), a member of avian retrovirus, also encodes a novel miRNA via canonical vmiRNA biosynthesis pathway. The vmiRNA has been designated as E (XSR) miRNA, since it is encoded by an virus specific region named exogenous element or XSR [50]. Similarly, Torque teno viruses (TTVs) a member of Anellovirus family also encodes miRNA, which inhibits the IFN signaling [51]. While studies of HIV-1 and Hepatitis $B$ virus showed no direct evidence for vmiRNAs expression, computational analysis has predicted five premiRNAs in HIV-1 and one pre-miRNA in by Hepatitis $B$ virus [52].

\section{miRNA modulating Hepatitis $\mathrm{C}$ virus infection}

Considerable evidences suggesting the role of miRNAs in modulating Hepatitis $\mathrm{C}$ virus (HCV) life cycle, infectivity and host defense mechanisms have opened a novel avenue for innovative therapeutic approaches for $\mathrm{HCV}$ infection. miR-122, which is abundantly expressed in liver cells, interacts with HCV genomic RNA and facilitates its replication in infected cells $[45,53]$. The interaction is mediated through binding of two copies of miR-122 to their respective seed map sites located within the $5^{\prime}$ UTR of the HCV genome [43, 45] The stable heterotrimeric interaction enhances HCV translation by promoting its association with ribosomes during the early initiation stage of translation [43]. Furthermore, miR-122 associated Argonaute proteins attached to the $5^{\prime}$ end of HCV genomic RNA protects the RNA from 5' exonuclease activity, specifically of the $5^{\prime}$ to $3^{\prime}$ exoribonuclease 1 (Xrn1) [54, 55]. Interestingly, miR-122 
interaction with the 5' UTR of HCV RNA produces a 3' overhang and masks the $5^{\prime}$ UTR, circumventing recognition by RNA helicase and ultimately reducing RNA decay [56]. Thus, miR-122 has a crucial role in enhancing HCV replication either by 5' UTR masking or other mechanisms [57]. Targeting miR-122 could be a novel approach for developing a therapy against chronic HCV infections and the miRNA can be employed as a biomarker of hepatic damage by the virus. The most promising example for miRNA based therapeutic approach is miravirsen, an oligonucleotide which has been demonstrated to inhibit the function of miR-122 [43].

Conversely, miR-199a, Let-7b, miR-448 and miR-196 are all implicated in suppressing HCV RNA replication [58-60]. miR-199a counteracts the action of miR-122 and represses HCV replication by binding to the seed map site within the $5^{\prime}$ UTR of the HCV genome just downstream of the second miR-122 binding site [58]. Let-7b, expressed in various tissues including liver and spleen, binds to the HCV RNA genome at various positions including the $5^{\prime}$ UTR and NS5B coding region leading to repression of $\mathrm{HCV}$ replication, possibly inducing conformational changes in the viral RNA genome [59]. miR-196 and miR-448 are also capable of directly binding to and interacting with the HCV RNA genome and exerting inhibitory effects on HCV replication [60]. Recently, miR-181c was reported to bind to the E1 and NS5A regions of the HCV genome and have a downregulating role in viral replication (Fig. 2) [8]. So, the alternative therapeutic approach could be upregulation of these miRNAs to suppress HCV replication.
In addition to the direct interaction with HCV RNA, some cellular miRNAs affect the HCV replication indirectly modulating replication-related pathways like Interferon (IFN)-mediated viral defense, Nuclear Factor-Kappa B (NF$\kappa \mathrm{B})$, Hemeoxygenase 1 (HMOX1) and lipid metabolism (Table 1) [43]. Interestingly, HCV infection up-regulates miR-21 and miR-130a expression, both of which negatively regulate their target genes known to trigger viral replication in cells, by decreasing HCV-mediated IFN type I (IFN-I) production and disrupting the process of viral entry, respectively [61, 62]. Up-regulation of miR- 21, and some other miRNAs like miR-134, miR-320c, and miR-483-5p has been shown to inhibit the NF-kB and PI3K-Akt pathways thereby suppressing anti-viral effect in HCV-infected patients [63, 64]. Also, miR-196 suppresses the antiviral effect on HCV by suppressing HMOX1 and miR279 inhibits $\mathrm{HCV}$ replication through regulation of lipid metabolism [43].

In addition, some miRNAs are implicated in complications related to $\mathrm{HCV}$ infection although their target and exact mechanism of action is still not clear. Upregulation of miR-276 promotes liver stenosis whereas down-regulation of miR-449a and miR-107, as well as up-regulation of miR-200c, supports hepatic fibrosis [43, 65]. Similarly, up-regulation of miR-155 and miR141 , as well as down-regulation of miR-152 and miR-491 (which are tumor suppressors), promotes hepatocellular carcinoma in HCV infection [43, 65]. miR-155, an indicator of hepatitis-induced hepatic damage, is over-expressed in the circulation of patients with chronic HCV infection [65]. Over-expression of miR-155 may inhibit apoptosis of

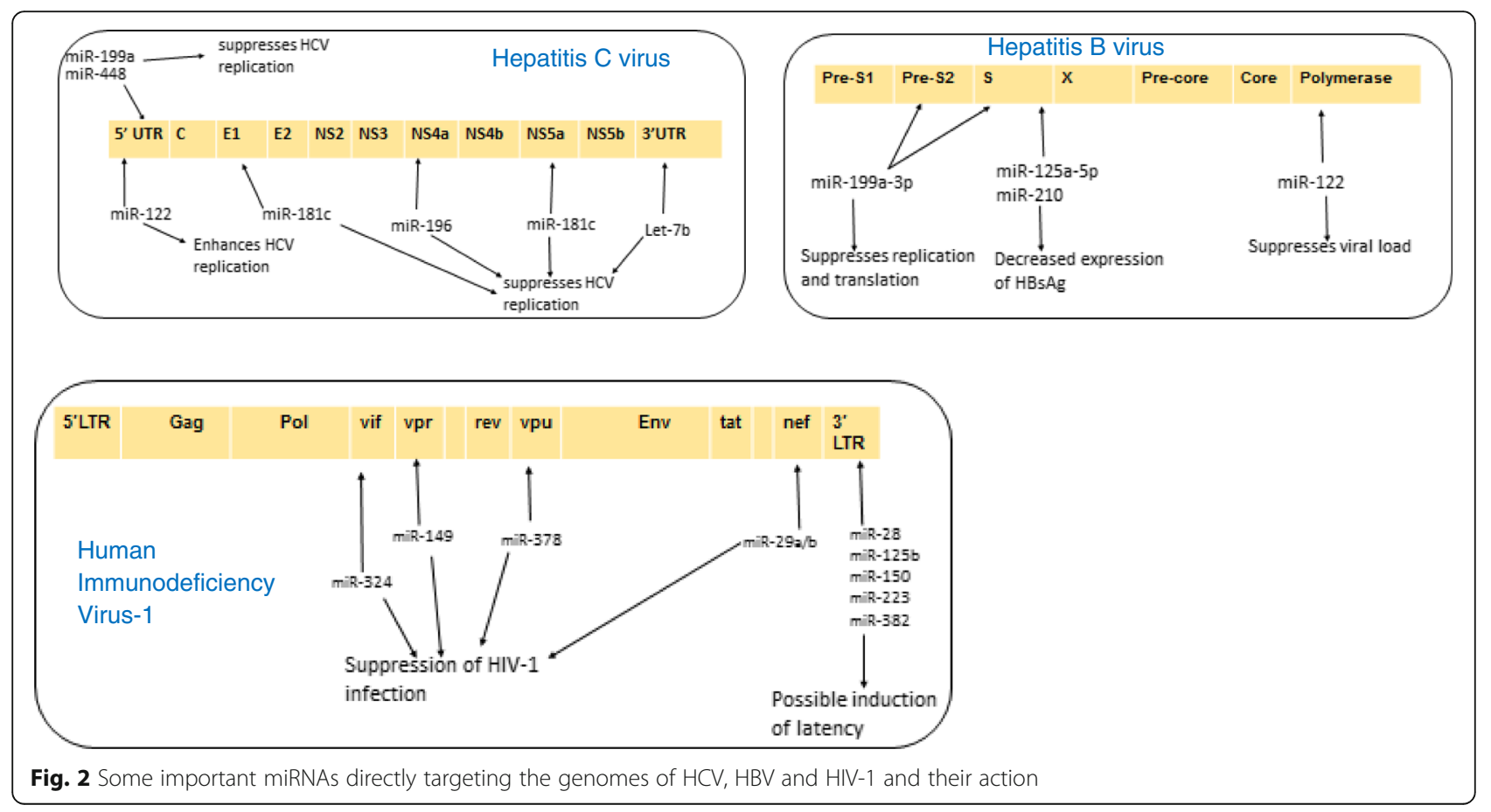


Table 1 Summary of some important miRNAs modulating Hepatitis C and B and HIV-1 infection by targeting host factors

\begin{tabular}{|c|c|c|}
\hline miRNAs & Targets & Actions \\
\hline \multicolumn{3}{|l|}{ Hepatitis $C$ virus } \\
\hline miR-21 & IFN1 & Suppress viral replication \\
\hline miR-130a & HCV entry & \\
\hline miR-21/miR-134/miR-320c/miR-483-5p & NFkB and PI3K-Akt & Inhibit NFkB and PI3K-Akt signaling pathway \\
\hline miR-196 & HMOX1 & Increase replication \\
\hline miR-279 & Lipid metabolism & Inhibit replication \\
\hline miR-155 & wnt signaling & Immune defense against the virus \\
\hline \multicolumn{3}{|l|}{ Hepatitis B virus } \\
\hline miR-122 & upregulation of $\mathrm{HMOX}_{1}$ & Decrease virus level in cell \\
\hline miR-501 & HBxIP & \\
\hline $\operatorname{miR}-372 / 373$ & NFIB & Promotes replication \\
\hline miR-155 & IFN1 & Suppress HBV disease pathogenesis \\
\hline \multicolumn{3}{|l|}{ Human Immunodeficiency Virus type 1} \\
\hline miR-27b & Cyclin T1 & Prevent the activation of CD4+ cells \\
\hline \multirow[t]{2}{*}{ miR-155 } & TLR3/Lymphocytes/DC & Reduces HIV-1 infection \\
\hline & 3' UTR of HDFs; LEDGF/p75, ADAM10, NUP 153 & Decrease HIV replication \\
\hline miR-146a & CXCR4 & Prevents HIV-1 entry \\
\hline miR-132 & MeCP-2 & Enhances HIV-1 infection \\
\hline miR-217 and miR-34a & SIRT1 & Enhances HIV-1 tat-mediated trans-activation \\
\hline miR-182 & NAMPT & Enhances HIV-1 tat-mediated trans-activation \\
\hline miR-34a & PNUTS & Promotes HIV-1 transcription \\
\hline miR-17/92 cluster and miR-20a & PCAF & Decrease susceptibility to HIV-1 infection \\
\hline
\end{tabular}

DC dendritic cells, SIRT1 Sirtuin 1, NAMPT Nicotinamide phosphoribosyltransferase, LEDGF Lens Epithelium-derived Growth factor, ADAM10 a disintegrin and metalloprotease, MeCP2 methyl CpG binding protein 2, HDF HIV dependency factors, nup153 Nuclear pore complex protein Nup153, HBx interacting protein, NFkB nuclear factor kappa B. IFNI Interferon I, HMOX1 Heme oxygenase 1, PNUTS phosphatase 1 nuclear-targeting subunit, PCAF p300-CREB binding protein associated factor

hepatocytes and promote cell proliferation by activating Wnt signaling leading to progression to hepatocellular carcinoma [65, 66]. Conversely, the suppression of miR155 may lead to cell cycle arrest in the G0 or G1 phase suggesting the positive role of miR-155 in immune defense against HCV infection [65]. Therefore, balanced expression of miR-155 is essential to retard the development of hepatocellular carcinoma and at the same time promote adequate cell cycle.

\section{miRNA modulating Hepatitis B virus infection}

Several cellular miRNAs modulate Hepatitis B virus (HBV) replication directly by binding to its transcripts (Fig. 2) or indirectly by targeting cellular factors, genes and signaling pathways related to HBV replication and pathogenesis (Table 1) [67]. The miRNAs shown to reduce HBV replication and the expression of HBV surface antigen (HBsAg) are miR-199a-3p, miR-210 and miR-125a-5p. Among them, miR-199a-3p targets HBsAg coding region and the pre-S region of the HBV genome, whereas miR-125a-5p binds to HBsAg mRNA leading to inhibition of its translation [46, 68]. miR-122 which enhances HCV replication, has opposite effect on HBV, where it upregulates HMOX1 to reduce HBV levels in cells. miR-122 also has cyclin G1-modulated antiHBV activity and directly affect the viral DNA polymerase [46]. These findings imply that Miravirsen targeting miR-122 will have opposite effect if used as therapeutics and other candidate miRNAs need to be considered.

Conversely, HBV can trigger changes in the expression of cellular miRNAs targeting negative regulators of HBV replication [69]. miR-501 which targets $\mathrm{HBx}$ interacting protein (HBxIP) and miR-372/373 which targets nuclear factor I B (NF-IB) of the host to promote viral replication are upregulated by HBV [70, 71]. HBx protein of HBV supports cellular proliferation by downregulating let-7a, which negatively regulates cellular proliferation partly through targeting signal transducer and activator of transcription 3 (STAT3) [69]. Furthermore, some miRNAs are involved in induction and suppression of HBV-related complications like hepatocellular carcinoma and cirrhosis. miR-155 by up-regulation of IFN-inducible genes suppresses HBV disease progression, whereas miR-548 by down-regulation of the host anti-viral response promotes HBV progression [46]. 


\section{miRNA modulating Human Immunodeficiency Virus infection}

Cellular miRNAs have been shown to restrict Human immunodeficiency virus type $1(\mathrm{HIV}-1)$ infection either by direct binding to the viral RNA or by indirect modulation of HIV Dependency Factors (HDFs) (reveiwed in ref [72]). CD4 ${ }^{+}$T-cells have been found to express some miRNAs which target mRNA of the viral genes (Fig. 2); negative regulatory factor (nef), viral protein $\mathrm{s}(\mathrm{vpr})$, viral infectivity factor (vif) and viral protein $U$ (vpu); and regulate host factors like Cyclin $\mathrm{T} 1$ protein and receptors or co-receptors needed for HIV entry [73-76]. Similarly other cells involved in the spread of HIV-1 within the body are also reported to express some miRNAs that defend against the virus [30].

The miRNAs that directly bind to the 3'UTR of HIV-1 RNA (Fig. 2) include miR-28, miR-125b, miR-150, miR223 and miR-382, which are highly expressed in resting CD4+T cells [73]. Activation of resting $\mathrm{CD}^{+}$T-cells (which results in more HIV replication) leads to downregulation of these miRNAs, which can be correlated with enhanced susceptibility to HIV-1 infection [73]. Other miRNAs target host factors and indirectly modulate HIV1 replication, susceptibility and latency in different cells (Table 1). miR-27b has been reported to inhibit the expression of Cyclin $\mathrm{T} 1$ in resting $\mathrm{CD}_{4}^{+} \mathrm{T}$-cells. Activation of CD4+ T-cells has been shown to down-regulate miR27b, subsequently up-regulating Cyclin T1. Up-regulation of Cyclin T1 results in enhanced HIV-1 susceptibility of CD4 ${ }^{+}$T-cells [77]. Also, miR-155 has been shown to regulate immune responses to HIV-1 infection by altering lymphocyte responses, inhibiting dendritic cell binding to HIV-1 and modulating TLR3 stimulation in macrophages leading to anti-HIV-1 effects [65]. miR-155 has been reported to target the 3' UTR of mRNAs of the HDFs; the lens epithelium-derived growth factor (LEDGF)/p75, ADAM10 (a disintegrin and metalloprotease 10) and nucleoporin (NUP153), and down-regulate their protein expression in primary macrophages [26]. Opposing the findings described above, Cullin et al. reported that HIV-1 neither encodes viral miRNAs nor strongly modulates cellular miRNA expression possibly due to extensive RNA secondary structures that block potential miRNA binding sites [78].

The miR-17/92 cluster and miR-20a are reported to target p300-CREB binding protein associated factor (PCAF) and make the cell less susceptible to HIV-1 infection, because PCAF has a role in tat acetylation (acetylated tat is transcriptionally more active) [79]. On the other hand, HIV-1 tends to suppress these miRNAs to upregulate PCAF expression and possibly increasing viral infectivity [80]. It has -been reported that expression of purine-rich element binding protein alpha (Pur- $\alpha)$, which facilitates HIV-1 transcription by binding tat protein and the TAR element, was significantly lower in monocytes than in monocyte-derived dendritic cells (DCs) [81]. Several of the cellular miRNAs (miR-15a, miR-15b, miR-16, miR20a, miR-93 and miR-106b) which target pur- $\alpha$ mRNA were overexpressed in monocytes. The correlation between low expression of pur- $\alpha$ and overexpression of these miRNAs indicates their role in decreasing susceptibility to HIV-1 infection [80, 82].

The interaction between HIV-1 RNA and proteins involved in the biogenesis of cellular miRNAs has also been reported. Silencing the genes for Drosha and Dicer showed increased viral replication in HIV-1-infected mononuclear cells even with latent infection [80]. Furthermore, HIV-1 gene expression was found to be negatively regulated by Argonaute or other miRNA related proteins by restricting the association of viral mRNA with polysomes [30]. Interestingly, silencing of Dicer has led to virus re-activation in peripheral blood mononuclear cells (PBMCs) isolated from HIV-1-infected patients undergoing suppressive combinational antiretroviral therapy (cART) [30].

On the other hand, some cellular miRNAs enhance HIV-1 replication by various mechanism. Over-expression of miR-132 down-regulates methyl CpG binding protein 2 (MeCP2) leading to enhancement of HIV-1 infection [76]. In addition, miR-217 and miR-34a, which have been reported to be up-regulated by tat exposure, bind to the 3' UTR of Sirtuin 1 (SIRT1) mRNA inhibiting its expression. Inhibition of SIRT1 which de-acetylates tat protein and regulates HIV-1 transcription, enhances HIV-1 tatmediated transactivation [83, 84]. Furthermore, a recent study revealed that down-regulation of nicotinamide phosphoribosyl transferase (NAMPT) by miR-182 led to decreased SIRT1 expression levels, which in turn enhanced HIV-1 Tat trans-activation [30, 72]. HIV-1 induces the expression of miR-34a, which promotes HIV-1 replication in CD4+ T cells. The other target of miR-34a is phosphatase 1 nuclear-targeting subunit (PNUTS), which negatively regulates HIV-1 transcription by inhibiting the assembly of cyclin T1 and CDK9 to block the transcription elongation [85].

\section{Role of miRNA in Dengue virus infection}

A group of researchers has reported that Dengue virus (DENV) infection down regulates host cellular miRNA elements, notably Dicer, Drosha, Ago1 and Ago2, to facilitate its replication. They found that the downregulation was mediated by interferon independent activity of the viral protein NS4B which interferes the dicing of precursor miRNA [86]. DENV infection significantly induces the expression of miR-146a, which facilitates viral replication by targeting TNF Receptor Associated Factor 6 (TRAF6) and dampening Interferon $\beta$ (IFN- $\beta$ ) production [87]. All four subtypes of DENV downregulate the miRNA-133a which suppresses viral replication 
possibly via interference with polypyrimidine tract binding protein (PTB) expression [88]. Yet, another miRNA namely miR-548g-3p also regulates the replication of DENV by targeting stem loop A (SLA) promoter element within the 5'UTR of DENV genome [89]. miRNA expression profiling followed by qRT-PCR validation has revealed that 4 miRNAs were upregulated and 2 were downregulated in dengue patients. miR-21-5p and miR$146 a-5 p$, which were functionally involved in inflammation and cell proliferation, were significantly different from the control groups indicating their high sensitivity and specificity as indicators of dengue infection. Further, these two miRNAs were correlated with number of leucocytes and neutrophils. These findings have suggested that miRNAs can also be employed as a diagnostic marker for DENV infection [90]. On the other hand, small viral RNA resembling miRNA found in mosquitos infected with DENV type 2 is reported to specifically target a viral gene and auto-regulates viral replication [91]. Incorporation of the miRNA recognition element (MRE) of miR-122 into the viral mRNA might have inhibitory effect on susceptibility to DENV infection in hepatic cells, rendering the virus safer for vaccine preparation [92].

\section{Role of miRNA in Herpes virus infections}

Most of the miRNAs encoded by Herpes simplex virus-1 (HSV-1) and Herpes simplex virus-2 (HSV-2) are expressed in latency and bind to latency-associated transcripts, underscoring their role in the development of latency during Herpes virus infection. The principal target for the latency-inducing miRNAs (e.g. miR-138) is the infected cell polypeptide 0 protein (ICP0), which induces lytic genes in neuron [93]. Similarly, host cell miR-155, miR-146a and miR-21 are up-regulated in B-cells when latently infected by Epstein Bar Virus (EBV) [94]. miR-155 mediated attenuation of NF- $\mathrm{kB}$ signaling in $\mathrm{B}$ lymphocytes by EBV is pivotal for the stable maintenance of EBV genome and immortalization of B lymphocytes during latency [95]. Conversely, EBV latent membrane protein 1 (LMP1) upregulates miR-155 expression by activation of NF-kB pathway [96]. So, the complex interaction among miR155, LMP1 and NF-kB signaling seems to control the immortalization and latency in EBV infection. Cytomegalovirus (CMV) infection has been reported to repress the expression of miR-100 and miR-101, which are linked to apoptosis [97]. Some oncogenic herpesviruses encode viral miRNA that mimic and exploit miR-155-mediated regulatory pathways for their own benefit. Kshv-miR-K1211, the viral functional ortholog of miR-155 encoded by Kaposi sarcoma-associated herpesvirus (KSHV), mimics miR-155 and utilizes its regulatory pathway to immortalize infected lymphocytes. KSHV-miR-12-11 attenuates TGF- $\beta$ via downregulation of SMAD5 [98]. The other miR-155 functional ortholog, miR-M4 encoded by Marek's disease chicken virus (MDV), may also be responsible for contributing to viral oncogenicity by down-regulating its target latent TGF- $\beta$-binding protein-1 (LTBP1), leading to reduced secretion of TGF- $\beta 1$. The resulting suppression of TGF- $\beta$ signaling might contribute to the activation of the oncogene c-Myc [65].

\section{Viral RNAi suppressors}

Viral RNAi suppressors (VSRs) are the virus encoded proteins that counteract against the RNA interference mediated by miRNA or other small RNA. Extensive research has been carried out in plant and insect VSRs, but only a few of them from mammalian viruses have been identified [99]. Some of the examples for mammalian VSRs are nucleocapsid protein ( $\mathrm{N}$ protein) of Corona viruses, VP35 protein from Ebola virus, tat protein of HIV, Core protein (C) of $\mathrm{HCV}$, nonstructural protein 1 (NS1) from Human influenza virus and B2 protein of Nodamura virus [100-104]. N protein of Corona viruses, via its double-stranded RNA binding activity, suppresses the short hairpin RNAs (shRNAs) or siRNAs from mammalian cells [100]. Similarly, VP35 protein from Ebola virus, which is an antagonist of IFN, suppresses RNAi interference by binding to dsRNA in human cells [101]. Whereas, HIV-1 tat and HCV core protein counteract the RNAi in human cell lines by downregulating the expression or activity of Dicer, which processes precursor hairpin structures [102, 103]. Function of tat as a suppressor of RNAi by suppressing the common pathway in small RNA maturation is conserved across plants as well as animals including human [105]. A novel viral motif, arginine rich motif (ARM) in the HIV-1 tat and rev has been shown to counteract the RNA interference mediated by Dicer [106].

Similarly, HIV-1 vpr has also been reported to alter the expression of Dicer. Trans-activating response element (TAR) of the HIV-1 transcript and miRNA were reported to be packaged in exosomes released from HIV-1-infected cells. The miRNA taken together with TAR might modulate uninfected cells, perhaps to increase their susceptibility to infection [30]. Contrastingly, HIV-1 tat, HTLV-1 Tax and BFV Tas are not capable of suppressing the RNA interference either by siRNA or miRNA in human cells [107]. NS1 protein, one of the virulent factor of Influenza virus $A$, has also demonstrated its RNAi suppressor activity in plants and animal cells [108]. In Nodamura virus, a small virus infecting mammalian as well as insect host, B2 protein binds to Dicer and prevent post Dicer activity of RNAi [109]. Yet another mammalian virus, Porcine Reproductive and Respiratory Syndrome Virus (PRRSV), has also been reported to suppress shRNA, dsRNA and miRNA mediated RNA interference. Furthermore, this virus also down regulates the expression of Ago-2 [104]. 


\section{Epigenetic control of miRNA by pathogens}

Many of the miRNA such as miR-9, miR-34, miR-137, miR-148, miR-124; are found to be controlled by epigenetic mechanism like DNA methylation and histone acetylation [110]. Conversely, some of the miRNAs modulate the epigenetics by controlling the epigenetic regulators such as methyl transferase, histone deacetylases and polycomb group genes [111]. Epigenetic changes of miRNA or tumor suppressor genes can be induced by pathogenic microorganism, leading to carcinogenesis. Aberrant methylation of miRNA genes (hsa-miR-124) in cervical cancer cell line caused by Human papilloma virus (HPV) has also been reported [112, 113]. On the other hand, Cryptosporidium parvum, a protozoal parasite, hijacks the histone deacetylase and NF-kB signaling pathway to suppress two host miRNA namely miR-424 and miR-503 in the epithelial cells [111]. Two of the miRNA namely miR-1 and miR-152 are reported to be involved in HBV pathogenesis via epigenetic control [114, 115]. miR-1 upregulates HBV transcription by enhancing farnesoid $\mathrm{X}$ receptor $\alpha$ expression but downregulates host cell cycle and cell proliferation by targeting histone deacetylase 4 and E2F transcription factor 5 [114]. Whereas, miR-152 reduces the levels of DNA methyltransferase 1 (DNMT1) in HBV induced Hepatocellular carcinoma by targeting 3'UTR of DNMT1 [115].

\section{Conclusion and perspectives}

Cellular miRNAs are important components of the host defense mechanism against viral infections. Many viruses are able to modulate cellular miRNA expression in host cells mostly in order to facilitate their survival, replication and pathogenesis. But, the overexpression of miRNA triggered by pathogens is not always correlated with their survival or pathogenesis and is sometimes cell or tissue specific. The exact mechanism(s) of modulation of host cellular miRNA by viruses and specific virulence factors is still unclear. Unraveling the molecular mechanism(s) of miRNA modulation by viral infections and vice versa will give direction to novel therapeutic approaches. Manipulation of miRNA, either by miRNA analogs or by inhibitors, could be a novel approach for developing therapies and prophylactic vaccines for various life-threatening viral infections. In addition, miRNAs may be exploited as biomarkers for laboratory diagnosis and prognosis. Some of the important cellular miRNA implicated in some viral infections are summarized in Table 1.

Some viruses encode for virus-specific miRNAs which are expressed in the host cell to subvert host defense and allow intracellular persistence. Understanding the specific functions of viral- miRNAs in the host-pathogen relationship will be another important step for targeting these miRNAs. All in all, miRNA may be a tool for diagnostic and therapeutic innovations against viral infections.

\begin{abstract}
Abbreviations
HBV: Hepatitis B virus; HCV: Hepatitis C virus; HDF: HIV dependency factor: HIV: Human Immunodeficiency Virus; HMOX1: Hemeoxygenase 1; IFN: Interferon; IncRNA: Long non-coding RNA; miR: microRNA; miRISC: microRNA-induced silencing complex; mRNA: Messenger RNA; NAMPT: Nicotinamide phosphoribosyl transferase; NF-kB: Nuclear Factor Kappa B; PCAF: p300-CREB binding protein associated factor; PrimiRNA: Primary miRNA; Pur-a: Purine-rich element binding protein alpha; RBP: RNA binding protein; RNA: Ribonucleic acid; SIRT1: Sirtuin 1; sncRNA: Small non-coding RNA; SRS: Suppressor of RNA silencing; TAR: Transactivation Response RNA; TLBP-1: TGF- $\beta$-binding protein-1; TLR: Toll-like receptor; UTR: Untranslated region
\end{abstract}

\section{Acknowledgement}

We are immensely grateful to all the faculty members of the Biomedical Science Program at Florida International University for their valuable suggestions and feedback. We are thankful for the Presidential Award provided by the graduate school of Florida International University.

\section{Funding}

Not applicable.

\section{Availability of data and materials \\ Not applicable.}

\section{Authors' contributions}

CRO: Manuscript outline, preparation of the draft manuscript, preparation of figures and table. Dr. MR: Critical reading and editing of the draft manuscript Dr. SMD: Critical reading and editing of the draft manuscript, preparation of conclusion table. Dr. RM: Critical reading and editing of the draft manuscript, writing of the abstract and the conclusion and perspectives section. Dr. NE$\mathrm{H}$ : Critical reading and editing of the draft manuscript, writing of the background section. All authors read and approved the final manuscript.

\section{Competing interests}

The authors declare that they have no competing interest.

Consent for publication

Not Applicable.

Ethical approval and consent to participate

Not Applicable.

\section{Author details}

${ }^{1}$ Department of Immunology, Herbert Wertheim College of Medicine, Florida International University, Florida, USA. ${ }^{2}$ Department of Cellular Biology and Pharmacology, Herbert Wertheim College of Medicine, Florida International University, Florida, USA.

Received: 21 July 2016 Accepted: 19 October 2016

Published online: 26 October 2016

\section{References}

1. Erdmann VA, Barciszewska MZ, Hochberg A, de Groot N, Barciszewski J. Regulatory RNAs. Cell Mol Life Sci. 2001;58(7):960-77.

2. Mattick JS, Makunin IV. Small regulatory RNAs in mammals. Hum Mol Genet. 2005;14 Spec No:R121-32.

3. Bartel DP. MicroRNAs: genomics, biogenesis, mechanism, and function. Cell. 2004:116(2):281-97.

4. Scott MS, Ono M. From snoRNA to miRNA: Dual function regulatory noncoding RNAs. Biochimie Elsevier. 2011;93(11):1987-92.

5. Wahid F, Shehzad A, Khan T, Kim YY. MicroRNAs: synthesis, mechanism, function, and recent clinical trials. Biochim Biophys Acta - Mol Cell Res. 2010;1803(11):1231-43.

6. He L, Hannon GJ. MicroRNAs: small RNAs with a big role in gene regulation. Nat Rev Genet. 2004:5(7):522-31.

7. Filipowicz W, Bhattacharyya SN, Sonenberg N. Mechanisms of posttranscriptional regulation by microRNAs: are the answers in sight? Nat Rev Genet. 2008;9(2):102-14.

8. Shrivastava $S$, Steele $R$, Ray $R$, Ray RB. MicroRNAs: role in hepatitis C virus pathogenesis. Genes Dis. 2015;2(1):35-45. 
9. Almeida MI, Reis RM, Calin GA. MicroRNA history: discovery, recent applications, and next frontiers. Mutat Res Mol Mech Mutagen. 2011;717(1-2):1-8.

10. Lee RC, Feinbaum RL, Ambros V. The C. elegans heterochronic gene lin-4 encodes small RNAs with antisense complementarity to lin-14. Cell. 1993; 75(5):843-54.

11. Reinhart BJ, Slack FJ, Basson M, Pasquinelli AE, Bettinger JC, Rougvie AE, et al. The 21-nucleotide let-7 RNA regulates developmental timing in Caenorhabditis elegans. Nature. 2000;403(6772):901-6.

12. Ambros V, Bartel B, Bartel DP, Burge CB, Carrington JC, Chen X, et al. A uniform system for microRNA annotation. RNA. 2003;9(3):277-9.

13. Lagos-Quintana M, Rauhut R, Lendeckel W, Tuschl T. Identification of novel genes coding for small expressed RNAs. Science. 2001;294(5543):853-8.

14. Kozomara A, Griffiths-Jones S. miRBase: annotating high confidence microRNAs using deep sequencing data. Nucleic Acids Res. 2014; 42(Database issue):D68-73.

15. Griffiths-Jones S. The microRNA registry. Nucleic Acids Res. 2004;32(Database issue):D109-11.

16. Dambal S, Shah M, Mihelich B, Nonn L. The microRNA-183 cluster: the family that plays together stays together. Nucleic Acids Res. 2015;43(15):7173-88.

17. Tang G-Q, Maxwell ES. Xenopus microRNA genes are predominantly located within introns and are differentially expressed in adult frog tissues via posttranscriptional regulation. Genome Res. 2008;18(1):104-12.

18. Melo CAM, SA. Biogenesis and Physiology of MicroRNAs. In: Fabbri M, editor. Non-coding RNAs and cancer. New York: Springer; 2014. p. 5-24

19. Lau NC, Lim LP, Weinstein EG, Bartel DP. An abundant class of tiny RNAs with probable regulatory roles in Caenorhabditis elegans. Science. 2001; 294(5543):858-62.

20. Calin GA, Sevignani C, Dumitru CD, Hyslop T, Noch E, Yendamuri S, et al. Human microRNA genes are frequently located at fragile sites and genomic regions involved in cancers. Proc Natl Acad Sci U S A. 2004;101(9):2999-3004.

21. He L, Thomson JM, Hemann MT, Hernando-Monge E, Mu D, Goodson S, et al. A microRNA polycistron as a potential human oncogene. Nature. 2005; 435(7043):828-33.

22. Lee Y, Ahn C, Han J, Choi H, Kim J, Yim J, et al. The nuclear RNase III Drosha initiates microRNA processing. Nature. 2003;425(6956):415-9.

23. Denli AM, Tops BB, Plasterk RH, Ketting RF, Hannon GJ. Processing of primary microRNAs by the Microprocessor complex. Nature. 2004;432(7014):231-5.

24. Okada C, Yamashita E, Lee SJ, Shibata S, Katahira J, Nakagawa A, et al. A high-resolution structure of the pre-microRNA nuclear export machinery. Science. 2009;326(5957):1275-9.

25. Lund E, Güttinger S, Calado A, Dahlberg JE, Kutay U. Nuclear export of microRNA precursors. Science. 2004;303(5654):95-8.

26. Yi R, Qin Y, Macara IG, Cullen BR. Exportin-5 mediates the nuclear export of pre-microRNAs and short hairpin RNAs. Genes Dev. 2003;17(24):3011-6.

27. Johanson TM, Lew AM, Chong MMW. MicroRNA-independent roles of the RNase III enzymes Drosha and Dicer. Open Biol. 2013;3(10):130144.

28. Wilson RC, Tambe A, Kidwell MA, Noland CL, Schneider CP, Doudna JA, et al. Dicer-TRBP complex formation ensures accurate mammalian MicroRNA biogenesis. Mol Cell Elsevier. 2015;57(3):397-407.

29. Fan $P$, Chen $Z$, Tian $P$, Liu W, Jiao $Y, X u e ~ Y$, et al. miRNA biogenesis enzyme drosha is required for vascular smooth muscle cell survival. Pesce $M$, editor. PLoS One. 2013;8(4):e60888.

30. Swaminathan G, Navas-Martín S, Martín-García J. MicroRNAs and HIV-1 infection: antiviral activities and beyond. J Mol Biol. 2014;426(6):1178-97.

31. Ruby JG, Jan CH, Bartel DP. Intronic microRNA precursors that bypass Drosha processing. Nature. 2007;448(7149):83-6.

32. Havens MA, Reich AA, Duelli DM, Hastings ML. Biogenesis of mammalian microRNAs by a non-canonical processing pathway. Nucleic Acids Res. 2012; 40(10):4626-40.

33. Pillai RS, Bhattacharyya SN, Filipowicz W. Repression of protein synthesis by miRNAs: how many mechanisms? Trends Cell Biol. 2007;17(3):118-26.

34. Bronevetsky Y, Ansel KM. Regulation of miRNA biogenesis and turnover in the immune system. Immunol Rev. 2013;253(1):304-16.

35. Ling $\mathrm{H}$, Fabbri $\mathrm{M}$, Calin GA. MicroRNAs and other non-coding RNAs as targets for anticancer drug development. Nat Rev Drug Discov. 2013;12(11):847-65.

36. Lytle JR, Yario TA, Steitz JA. Target mRNAs are repressed as efficiently by microRNA-binding sites in the 5' UTR as in the 3' UTR. Proc Natl Acad Sci U S A. 2007;104(23):9667-72.

37. Ameres SL, Horwich MD, Hung J-H, Xu J, Ghildiyal M, Weng Z, et al. Targe RNA-directed trimming and tailing of small silencing RNAs. Science. 2010; 328(5985):1534-9.
38. Vasudevan S, Steitz JA. AU-rich-element-mediated upregulation of translation by FXR1 and Argonaute 2. Cell. 2007;128(6):1105-18.

39. Vasudevan S, Tong Y, Steitz JA. Switching from repression to activation: microRNAs can up-regulate translation. Science. 2007:318(5858):1931-4.

40. Eulalio A, Schulte L, Vogel J. The mammalian microRNA response to bacterial infections. RNA Biol. 2012:9(6):742-50.

41. Skalsky RL, Cullen BR. Viruses, microRNAs, and host interactions. Annu Rev Microbiol Annual Reviews. 2010;64:123-41.

42. Staedel C, Darfeuille F. MicroRNAs and bacterial infection. Cell Microbiol. 2013;15(9):1496-507.

43. Li X, Yang W, Ye W, Jin L, He J, Lou L. microRNAs: novel players in hepatitis C virus infection. Clin Res Hepatol Gastroenterol. 2014;38(6):664-75.

44. Bogerd HP, Skalsky RL, Kennedy EM, Furuse Y, Whisnant AW, Flores O, et al. Replication of many human viruses is refractory to inhibition by endogenous cellular microRNAs. J Virol American Society for Microbiology (ASM). 2014;88(14):8065-76.

45. Jopling CL, Yi M, Lancaster AM, Lemon SM, Sarnow P. Modulation of hepatitis C virus RNA abundance by a liver-specific MicroRNA. Science. 2005; 309(5740):1577-81.

46. Kitab B, Alj HS, Ezzikouri S, Benjelloun S. MicroRNAs as important players in host-hepatitis B virus interactions. J Clin Transl Hepatol. 2015;3(2):149-61.

47. Grundhoff A, Sullivan CS. Virus-encoded microRNAs. Virology. 2011;411(2):325-43.

48. Kincaid RP, Burke JM, Sullivan CS. RNA virus microRNA that mimics a B-cell oncomiR. Proc Natl Acad Sci U S A. 2012;109(8):3077-82.

49. Whisnant AW, Kehl T, Bao Q, Materniak M, Kuzmak J, Löchelt M, et al. Identification of novel, highly expressed retroviral microRNAs in cells infected by bovine foamy virus. J Virol American Society for Microbiology. 2014:88(9):4679-86.

50. Yao $Y$, Smith LP, Nair V, Watson M. An avian retrovirus uses canonical expression and processing mechanisms to generate viral MicroRNA. J Virol American Society for Microbiology. 2014;88(1):2-9.

51. Kincaid RP, Burke JM, Cox JC, de Villiers E-M, Sullivan CS, Leppik L, et al. A human torque teno virus encodes a MicroRNA that inhibits interferon signaling. Cullen BR, editor. PLoS Pathog. 2013;9(12):e1003818.

52. Bennasser $Y$, Le S-Y, Yeung ML, Jeang K-T. HIV-1 encoded candidate microRNAs and their cellular targets. Retrovirology BioMed Central. 2004;1(1):43.

53. Randall G, Panis M, Cooper JD, Tellinghuisen TL, Sukhodolets KE, Pfeffer S, et al. Cellular cofactors affecting hepatitis $C$ virus infection and replication. Proc Natl Acad Sci U S A. 2007;104(31):12884-9.

54. Shimakami T, Yamane D, Jangra RK, Kempf BJ, Spaniel C, Barton DJ, et al. Stabilization of hepatitis C virus RNA by an Ago2-miR-122 complex. Proc Natl Acad Sci. 2012;109(3):941-6.

55. Li Y, Masaki T, Yamane D, McGivern DR, Lemon SM. Competing and noncompeting activities of miR-122 and the $5^{\prime}$ exonuclease Xrn1 in regulation of hepatitis C virus replication. Proc Natl Acad Sci. 2013;110(5):1881-6.

56. Machlin ES, Sarnow P, Sagan SM. Masking the $5^{\prime}$ terminal nucleotides of the hepatitis $C$ virus genome by an unconventional microRNA-target RNA complex. Proc Natl Acad Sci. 2011;108(8):3193-8.

57. Masaki T, Arend KC, Li Y, Yamane D, McGivern DR, Kato T, et al. miR-122 stimulates hepatitis $C$ virus RNA synthesis by altering the balance of vira RNAs engaged in replication versus translation. Cell Host Microbe. 2015; 17(2):217-28.

58. Murakami Y, Aly HH, Tajima A, Inoue I, Shimotohno K. Regulation of the hepatitis C virus genome replication by miR-199a. J Hepatol. 2009;50(3):453-60.

59. Cheng J-C, Yeh Y-J, Tseng C-P, Hsu S-D, Chang Y-L, Sakamoto N, et al. Let$7 \mathrm{~b}$ is a novel regulator of hepatitis C virus replication. Cell Mol Life Sci. 2012; 69(15):2621-33.

60. Lagos-Quintana M, Rauhut R, Yalcin A, Meyer J, Lendeckel W, Tuschl T. Identification of tissue-specific microRNAs from mouse. Curr Biol. 2002. 12(9):735-9.

61. Chen Y, Chen J, Wang H, Shi J, Wu K, Liu S, et al. HCV-induced miR-21 contributes to evasion of host immune system by targeting MyD88 and IRAK1. PLoS Pathog. 2013;9(4):e1003248

62. Bhanja Chowdhury J, Shrivastava S, Steele R, Di Bisceglie AM, Ray R, Ray RB. Hepatitis $C$ virus infection modulates expression of interferon stimulatory gene IFITM1 by upregulating miR-130A. J Virol. 2012;86(18):10221-5.

63. Shwetha S, Gouthamchandra K, Chandra M, Ravishankar B, Khaja MN, Das S. Circulating miRNA profile in HCV infected serum: novel insight into pathogenesis. Sci Rep. 2013;3:1555.

64. Marquez RT, Wendlandt E, Galle CS, Keck K, McCaffrey AP. MicroRNA-21 is upregulated during the proliferative phase of liver regeneration, targets 
Pellino-1, and inhibits NF- B signaling. AJP Gastrointest Liver Physiol. 2010; 298(4):G535-41.

65. Zeng F-R, Tang L-J, He Y, Garcia RC. An update on the role of miRNA-155 in pathogenic microbial infections. Microbes Infect. 2015;17(9):613-21.

66. Zhang Y, Wei W, Cheng N, Wang K, Li B, Jiang X, et al. Hepatitis C virusinduced up-regulation of microRNA-155 promotes hepatocarcinogenesis by activating Wnt signaling. Hepatology. 2012;56(5):1631-40.

67. Thirion $M$, Ochiya T. Roles of microRNAs in the hepatitis B virus infection and related diseases. Viruses. 2013;5(11):2690-703.

68. Zhang G, Li Y, Zheng S, Liu M, Li X, Tang H. Suppression of hepatitis B virus replication by microRNA-199a-3p and microRNA-210. Antiviral Res. 2010; 88(2):169-75.

69. Wang Y, Lu Y, Toh ST, Sung W-K, Tan P, Chow P, et al. Lethal-7 is downregulated by the hepatitis $B$ virus $x$ protein and targets signal transducer and activator of transcription 3. J Hepatol. 2010:53(1):57-66.

70. Jin J, Tang S, Xia L, Du R, Xie H, Song J, et al. MicroRNA-501 promotes HBV replication by targeting HBXIP. Biochem Biophys Res Commun. 2013;430(4): 1228-33.

71. Guo H, Liu H, Mitchelson K, Rao H, Luo M, Xie L, et al. MicroRNAs-372/373 promote the expression of hepatitis $B$ virus through the targeting of nuclear factor I/B. Hepatology. 2011;54(3):808-19.

72. Pilakka-Kanthikeel S, Nair MP. Interaction of drugs of abuse and microRNA with HIV: a brief review. Front Microbiol. 2015;6:967.

73. Huang J, Wang F, Argyris E, Chen K, Liang Z, Tian H, et al. Cellular microRNAs contribute to HIV-1 latency in resting primary CD4+ T lymphocytes. Nat Med. 2007;13(10):1241-7.

74. Hariharan M, Scaria V, Pillai B, Brahmachari SK. Targets for human encoded microRNAs in HIV genes. Biochem Biophys Res Commun. 2005;337(4):1214-8.

75. Nathans R, Chu C-Y, Serquina AK, Lu C-C, Cao H, Rana TM. Cellular microRNA and P bodies modulate host-HIV-1 interactions. Mol Cell. 2009; 34(6):696-709.

76. Chiang K, Liu H, Rice AP. miR-132 enhances HIV-1 replication. Virology. 2013; 438(1):1-4.

77. Chiang K, Sung T-L, Rice AP. Regulation of cyclin T1 and HIV-1 Replication by microRNAs in resting CD4+ T lymphocytes. J Virol. 2012;86(6):3244-52.

78. Whisnant AW, Bogerd HP, Flores O, Ho P, Powers JG, Sharova N, et al. Indepth analysis of the interaction of HIV-1 with cellular microRNA biogenesis and effector mechanisms. MBio American Society for Microbiology (ASM). 2013;4(2):e000193.

79. Brès $V$, Tagami $H$, Péloponèse $J-M$, Loret $E$, Jeang $K-T$, Nakatani $Y$, et al. Differential acetylation of Tat coordinates its interaction with the coactivators cyclin T1 and PCAF. EMBO J European Molecular Biology Organization. 2002;21(24):6811-9.

80. Triboulet R, Mari B, Lin Y-L, Chable-Bessia C, Bennasser Y, Lebrigand K, et al. Suppression of microRNA-silencing pathway by HIV-1 during virus replication. Science. 2007;315(5818):1579-82.

81. Shen C-J, Jia Y-H, Tian R-R, Ding M, Zhang C, Wang J-H. Translation of Pur-a is targeted by cellular miRNAs to modulate the differentiation-dependent susceptibility of monocytes to HIV-1 infection. FASEB J. 2012;26(11):4755-64

82. Deng L, de la Fuente C, Fu P, Wang L, Donnelly R, Wade JD, et al. Acetylation of HIV-1 Tat by CBP/P300 increases transcription of integrated HIV-1 genome and enhances binding to core histones. Virology. 2000;277(2):278-95.

83. Zhang H-S, Wu T-C, Sang W-W, Ruan Z. MiR-217 is involved in Tat-induced HIV-1 long terminal repeat (LTR) transactivation by down-regulation of SIRT1. Biochim Biophys Acta - Mol Cell Res. 2012;1823(5):1017-23.

84. Zhang H-S, Chen X-Y, Wu T-C, Sang W-W, Ruan Z. MiR-34a is involved in Tat-induced HIV-1 long terminal repeat (LTR) transactivation through the SIRT1/NFKB pathway. FEBS Lett. 2012;586(23):4203-7.

85. Kapoor R, Arora S, Ponia SS, Kumar B, Maddika S, Banerjea AC, et al. The miRNA miR-34a enhances HIV-1 replication by targeting PNUTS/PPP1R10, which negatively regulates HIV-1 transcriptional complex formation. Biochem J Portland Press Limited. 2015;470(3):293-302.

86. Kakumani PK, Ponia SS, S RK, Sood V, Chinnappan M, Banerjea AC, et al. Role of RNA Interference (RNAi) in Dengue Virus Replication and Identification of NS4B as an RNAi Suppressor. J Virol. 2013;87(16):8870-83.

87. Wu $S$, He L, Li Y, Wang T, Feng $L$, Jiang $L$, et al. miR-146a facilitates replication of dengue virus by dampening interferon induction by targeting TRAF6. J Infect. 2013;67(4):329-41.

88. Castillo JA, Castrillón JC, Diosa-Toro M, Betancur JG, St Laurent G, Smit JM, et al. Complex interaction between dengue virus replication and expression of miRNA-133a. BMC Infect Dis. 2016;16:29.
89. Wen $W$, He Z, Jing $Q, H u ~ Y$, Lin $C$, Zhou R, et al. Cellular microRNAmiR-548g-3p modulates the replication of dengue virus. J Infect. 2015; 70(6):631-40

90. Ouyang X, Jiang X, Gu D, Zhang Y, Kong SK, Jiang C, et al. Dysregulated Serum MiRNA Profile and Promising Biomarkers in Dengue-infected Patients. Int J Med Sci. 2016;13(3):195-205.

91. Hussain M, Asgari S. MicroRNA-like viral small RNA from Dengue virus 2 autoregulates its replication in mosquito cells. Proc Natl Acad Sci. 2014; 111(7):2746-51

92. Lee T-C, Lin Y-L, Liao J-T, Su C-M, Lin C-C, Lin W-P, et al. Utilizing liverspecific microRNA-122 to modulate replication of dengue virus replicon. Biochemical and Biophysical Research Communications. 2010;396

93. Lukonis CJ, Weller SK. The herpes simplex virus type 1 transactivator ICPO mediates aberrant intracellular localization of the viral helicase/primase complex subunits. Virology. 1996;220(2):495-501.

94. Frappier L. Regulation of herpesvirus reactivation by host microRNAs. J Virol. 2015;89(5):2456-8.

95. Lu F, Weidmer A, Liu C-G, Volinia S, Croce CM, Lieberman PM. Epstein-Barr Virus-Induced miR-155 Attenuates NF- B Signaling and Stabilizes Latent Virus Persistence. J Virol. 2008;82(21):10436-43.

96. Gatto G, Rossi A, Rossi D, Kroening S, Bonatti S, Mallardo M. Epstein-Barr virus latent membrane protein 1 trans-activates miR-155 transcription through the NF-kappaB pathway. Nucleic Acids Res. 2008;36(20):6608-19.

97. Yang $G$, Gong $Y$, Wang $Q$, Wang $Y$, Zhang $X$. The role of miR-100mediated Notch pathway in apoptosis of gastric tumor cells. Cell Signal. 2015;27(6):1087-101.

98. Liu Y, Sun R, Lin X, Liang D, Deng Q, Lan K. Kaposi's sarcoma-associated herpesvirus-encoded microRNA miR-K12-11 attenuates transforming growth factor beta signaling through suppression of SMAD5. J Virol. 2012;86(3): 1372-81.

99. Anand A, Mukherjee SK, Sanan-Mishra N. Tools for pathogenicity: virus encoded RNA silencing suppressors.

100. Cui L, Wang H, Ji Y, Yang J, Xu S, Huang X, et al. The nucleocapsid protein of coronaviruses acts as a viral suppressor of RNA silencing in mammalian cells. J Virol. 2015;89(17):9029-43.

101. Haasnoot J, de Vries W, Geutjes E-J, Prins M, de Haan P, Berkhout B. The Ebola virus VP35 protein is a suppressor of RNA silencing. PLoS Pathog. 2007:3(6):e86.

102. Bennasser Y, Le S-Y, Benkirane M, Jeang K-T, Baulcombe DC, Molnar A, et al. Evidence that HIV-1 encodes an siRNA and a suppressor of RNA silencing. Immunity. 2005;22(5):607-19.

103. Chen W, Zhang Z, Chen J, Zhang J, Zhang J, Wu Y, et al. HCV core protein interacts with Dicer to antagonize RNA silencing. Virus Res. 2008;133(2):250-8.

104. Lin M. Discovery and roles of virus-encoded RNA silencing suppressors. SM Virol. 2016;1(2):1007.

105. Qian S, Zhong X, Yu L, Ding B, de Haan P, Boris-Lawrie K. HIV-1 Tat RNA silencing suppressor activity is conserved across kingdoms and counteracts translational repression of HIV-1. Proc Natl Acad Sci U S A. 2009;106(2):605-10.

106. Ponia S, Arora S, Kumar B, Banerjea AC, Neduva V, Russell R, et al. Arginine rich short linear motif of HIV-1 regulatory proteins inhibits Dicer dependent RNA interference. Retrovirology BioMed Central. 2013;10(1):97.

107. Lin J, Cullen BR. Analysis of the interaction of primate retroviruses with the human RNA interference machinery. J Virol. 2007:81 (22):12218-26.

108. Bivalkar-Mehla S, Vakharia J, Mehla R, Abreha M, Kanwar JR, Tikoo A, et al. Viral RNA silencing suppressors (RSS): novel strategy of viruses to ablate the host RNA interference (RNAi) defense system. Virus Res. 2011; 155(1):1-9.

109. Sullivan CS, Ganem D. A virus-encoded inhibitor that blocks RNA interference in mammalian cells. J Virol. 2005;79(12):7371-9.

110. Sato F, Tsuchiya S, Meltzer SJ, Shimizu K. MicroRNAs and epigenetics. FEBS J. 2011;278(10):1598-609.

111. Zhou R, Gong A-Y, Chen D, Miller RE, Eischeid AN, Chen X-M, et al. Histone deacetylases and NF-kB signaling coordinate expression of CX3CL1 in epithelial cells in response to microbial challenge by suppressing miR-424 and miR-503. Zilberstein D, editor. PLoS One. 2013; 8(5):e65153.

112. Wilting SM, van Boerdonk RAA, Henken FE, Meijer CJLM, Diosdado B, Meijer $\mathrm{GA}$, et al. Methylation-mediated silencing and tumour suppressive function of hsa-miR-124 in cervical cancer. Mol Cancer. 2010;9:167. 
113. Jiménez-Wences $H$, Peralta-Zaragoza O, Fernández-Tilapa G. Human papilloma virus, DNA methylation and microRNA expression in cervical cancer (Review). Oncol Rep Spandidos Publications. 2014; 31(6):2467-76.

114. Zhang X, Zhang E, Ma Z, Pei R, Jiang M, Schlaak JF, et al. Modulation of hepatitis $B$ virus replication and hepatocyte differentiation by MicroRNA-1. Hepatology. 2011;53(5):1476-85.

115. Huang J, Wang Y, Guo Y, Sun S. Down-regulated microRNA-152 induces aberrant DNA methylation in hepatitis B virus-related hepatocellular carcinoma by targeting DNA methyltransferase 1. Hepatology. 2010;52(1):60-70.

Submit your next manuscript to BioMed Central and we will help you at every step:

- We accept pre-submission inquiries

- Our selector tool helps you to find the most relevant journal

- We provide round the clock customer support

- Convenient online submission

- Thorough peer review

- Inclusion in PubMed and all major indexing services

- Maximum visibility for your research

Submit your manuscript at www.biomedcentral.com/submit
Biomed Central 